\title{
Synthesis and Characterization of paramagnetic (poly styrene-co- acrylamide) hydrogel: used as adsorbent for removal of pesticide (chlorpyrifos)
}

\author{
Anita Bind And Alka Tiwari* \\ Research Centre, Department of Chemistry, Govt.V.Y.T.PG. Autonomous College Durg (C.G.) - 491001 India.
}

\begin{abstract}
The sorption potential of paramagnetic poly styrene-co-acrylamide hydrogel was investigated for the removal of pesticide (Chlorpyrifos) from aqueous solution. The maximum uptake of pesticide was obtained at pH 4 in 60 minute at room temperature $\left(25^{\circ} \mathrm{C}\right)$. In batch mode of adsorption, the influence of $\mathrm{pH}$, concentration, adsorbent dose and in column mode, bed height, flow rate and initial concentration were studied on adsorptive removal of pesticide. The equilibrium experimental results were analyzed in terms of Langmuir and Freundlich models. Equilibrium data fitted very well to the Langmuir model. In the column method, the BDST model was used to obtain an empirical relationship for the design of adsorption column. The adsorbent "paramagnetic poly styrene-co-acrylamide hydrogel" was characterized by TEM, XRD and FTIR analysis respectively.
\end{abstract}

Keywords: Nano particles, Pesticide, Isotherm, Equilibrium.

\section{Introduction}

The widespread use and disposal of pesticides by farmers, institutions and the general public provide many possible sources of pesticides in the environment. Following release into the environment, pesticides may have many different fates. Pesticides which are sprayed can move through the air and may eventually end up in other parts of the environment, such as in soil or water. Pesticides which are applied directly to the soil may be washed off the soil into nearby bodies of surface water or may percolate through the soil to lower soil layers and groundwater. Pesticides which are injected into the soil may also be subject to the latter two fates. The application of pesticides directly to bodies of water for weed control, or indirectly as a result of leaching from boat paint, runoff from soil or other routes, may lead not only to build up of pesticides in water, but also may contribute to air levels through evaporation. Organic pesticides such as parathion-methyl, chlorpyrifose, carbaryl and carbofuran are highly toxic organic pollutants and are widely used in cotton, sugar cane, rice, citrus and other crops in India. Due to their wide applications, these pesticides are often detected in fresh water exceeding permissible levels and cause harmful impact on human health [1]. The pesticides form a strong class of water pollutants as they are mostly non-biodegradable and carcinogenic in nature [2-3]. Therefore toxicity of pesticides and their degradation products are making these chemical substances a potential hazard by contaminating the environment [4]. The toxic effects of pesticides cause serious problems in both the environment and human beings [5]. The transport and destination of pesticides involve complex mechanisms that are influenced by many processes including volatization, leaching, adsorption, chemical and biological decomposition [6].Among pesticides, organophosphorus pesticides $\left(\mathrm{OPP}_{\mathrm{S}}\right)$ are most commonly used [7]. Chlorpyrifos is a broad spectrum orgnophosphorus insecticide, widely used for crop protection and mosquito control [8]. Chlorpyrifos O, O-Diethyle-O-(3,5,6-trichloro-2 pyridylphosphorothiate (C9H11Cl3NO3PS) an oraganophosphate insecticide, is one of the most widely used active ingredients for pest control products worldwide [9-10].

Waste water treatment methods include precipitation, coagulation, floatation, sedimentation, filtration, membrane process, electrochemical techniques, ion exchange, biological process and chemical reactions; each method has its own merits and limitations in applications. Recent attention of the scientists has been devoted to the study of different types of low cost materials such as fertilizer waste [11-13] walnut shell [14] bagases [15] corn wood [16], bleaching earth[17] red mud [18], gas concrete material [19],industrial waste [20]. Nanocomposite hydrogels are among the most potential adsorbents for the removal of toxic pesticides from aqueous solutions because of the excellent mechanical and thermal properties as well as higher surface area and hydrodynamic radius for nano composite hydrogels in comparison with pure polymer hydrogels [22-23]. Super paramagnetic iron oxide nano particles [mostly magnetite $\left(\mathrm{Fe}_{3} \mathrm{O}_{4}\right)$ or maghemite $\left(\gamma-\mathrm{Fe}_{2} \mathrm{O}_{3}\right)$ ] with tailored surface chemistry have been widely used experimentally for numerous applications. These magnetic nano particles if incorporated in the co-polymeric matrix "poly styrene-co-acrylamide" by insitu method may be useful and effective in adsorbing the toxic pollutant due to the large surface area to volume ratio. All these application require the high magnetization value and size of magnetic nano particles smaller than $100 \mathrm{~nm}$. 
Synthesis and Characterization of paramagnetic (poly styrene-co-acrylamide) hydrogel: used as..

Thus in the present study by making the use of combined property "magnetism and adsorption", the effective adsorbent "poly styrene-co-acrylamide" has been synthesized and used as adsorbent for removal of pesticide "Chlorpyrifos" from water.

\section{Materials and methods}

2.1 Material: The monomer acrylamide, styrene, N, N- methylene- bis -acrylamide (cross-linker), potassium per sulphate (initiator), anhydrous ferric chloride and ferrous chloride tetra hydrate were purchased from Molychem, Mumbai, India. Double distilled water was used throughout the experiment.

\subsubsection{Synthesis of Super paramagnetic PSAM Hydrogel:}

To a mixture of styrene and acrylamide in 1:1 ratio, the cross linker ( $\mathrm{N}, \mathrm{N}$-methylene-bis-acrylamide) and initiator (potassium per sulphate) were added and the mixture was heated at $70^{\circ} \mathrm{C}$ in an electric oven for $1 \mathrm{hr}$. The co-polymeric hydrogel so formed was washed with distilled water and cut into small uniform pieces. For insitu magnetization, these pieces were kept in an aqueous solution of ferrous chloride and ferric chloride (in 1:2 ratio) for $24 \mathrm{hr}$. followed by the addition of concentrated ammonia solution and allowed to stand over night. The nano iron oxide loaded co-polymeric hydrogel was then washed thoroughly with distilled water, dried, crushed into a fine powder and stored.

\subsubsection{Preparation of stock solution:}

The stock solution of Chlorpyrifos of $1000 \mathrm{mg} \mathrm{dm}^{-3}$ concentration was prepared by dissolving $0.1 \mathrm{~g}$ of pesticide in $100 \mathrm{ml}$ of alcohol. Working solutions were prepared for adsorption experiments by diluting the stock solution.

\section{Synthesis of Gold nano particles}

Gold nano particles (Au@citrate) with an average diameter of 10-20 nm were synthesized by the reduction of $\mathrm{HAuCl} 4$. $3 \mathrm{H} 2 \mathrm{O}$ with trisodium citrate [24]. In this method $10 \mathrm{Ml}$ of $5 \mathrm{mM} \mathrm{HAuCl} 4.3 \mathrm{H} 2 \mathrm{O}$ was diluted with $150 \mathrm{~mL}$ distilled water and heated until it begins to boil. An amount measuring $10 \mathrm{~mL}$ of $0.5 \%$ trisodium citrate solution was added and continued heating until the solution turned wine red .It was cooled under ambient condition.

\section{Adsorption experiment}

For batch adsorption, a fixed amount of adsorbent was shaken with different volumes of pesticide solution at fixed $\mathrm{pH}$ and time in an orbital shaker. The concentration of pesticide before and after adsorption was determined spectrophotomatrically (Cary $60 \mathrm{UV} / \mathrm{Vis}$ spectrophotometer) by following equation:

$$
\begin{aligned}
& \text { Sorption degre }=\frac{C e-C 0}{C e} \times 1 \\
& \text { Sorption capacity }=\frac{C_{e}-C_{0}}{m_{s}}
\end{aligned}
$$

where $\mathrm{C}_{0}$ and $\mathrm{C}_{\mathrm{e}}(\mathrm{mg} / \mathrm{mL})$ are initial and equilibrium concentration of chlorpyrifos solutions respectively, $\mathrm{V}_{\text {sol }}$ $(\mathrm{L})$ is the volume of the chlorpyrifos solution subjected to sorption and $\mathrm{m}$ sorbent $(\mathrm{g})$ is the weight of sorbent.

\section{Fixed bed column studies}

For fixed bed column sorption, a column of $10 \mathrm{~cm}$ height and $1 \mathrm{~cm}$ diameter was filled with the magnetic adsorbent without gaps and then a known concentration of chlorpyrifos solution was allowed to pass through the bed at a constant flow rate in down flow manner. The pesticide solution was collected at different time intervals and its concentration was determined spectrophotomatrically.

The total amount of pesticide sent to the column $\left(\mathrm{m}_{\text {total }}\right)$ was calculated by the following equation:

$m_{\text {total }}=\frac{C_{0} Q t_{\text {total }}}{1000}$

where $\mathrm{C}_{0}$ influent concentration, $\mathrm{Q}$ and $\mathrm{t}_{\text {total }}$ are the influent flow rate and exhaustion time respectively. The adsorbed amount of pesticide in column study was determined by the following equation-

$$
\text { removal\% }=\frac{q_{\text {total }}}{m_{\text {total }}} X 100
$$

where $\mathrm{q}_{\text {total }}$ is sorbed quantity of adsorbate and $\mathrm{m}_{\text {total }}$ is the total amount of adsorbate sent to the column. 


\section{Results and discussion}

6.1. Characterization of adsorbent: The adsorbent "Super paramagnetic PSAM hydrogel" was characterized by FTIR, and TEM analysis.

FTIR Analysis: FTIR spectra of adsorbent were recorded using Varian Vertex FTIR Spectrometer (UGC-DAE, Indore, INDIA).

\section{TEM Analysis:}

The average particle size, size distribution and morphology of iron oxide nano particles were examined using a TECNAI-G20 TEM at a voltage of 200kv. The solvent dispersion of the particles were drop- cast onto a carbon coated copper grid and the grid was air dried at ambient conditions $\left(25 \pm 10{ }^{\circ} \mathrm{C}\right)$ before loading onto the microscope (AIIMS, New Delhi, INDIA).

(Fig. 1)

\subsection{FTIR Analysis:}

Fourier Transform Infrared spectroscopy (FTIR) of super paramagnetic Poly styrene-co-acrylamide Hyrdogel, as shown in figure 3 , indicated the peak at $2930.55 \mathrm{~cm}^{-}{ }^{-}$, assigned to C-H bond of methylene group ,at $3050-3000 \mathrm{~cm}^{-}{ }^{-}$, due to Ar-H stretching, $1600 \mathrm{~cm}^{-}{ }^{1}(\mathrm{v}), 1580 \mathrm{~cm}^{-}{ }^{-}(\mathrm{v})$, and $1450 \mathrm{~cm}^{-1}(\mathrm{~m})$, due to $\mathrm{C}=\mathrm{C}$ stretching respectively. Absorption peaks due to acrylamide were observed at $1725.16 \mathrm{~cm}^{-1}$, and $1652.76 \mathrm{~cm}^{-1}$ due to $\mathrm{C}=\mathrm{O}$, and $\mathrm{N}-\mathrm{H}$, stretching of the acrylamide $\left(-\mathrm{CONH}_{2}\right)$ group respectively and at $3449.24 \mathrm{~cm}^{-1}$ due to $-\mathrm{NH}_{2}$ stretching of $\left(-\mathrm{CONH}_{2}\right)$ group . The characteristic peak at $554.39 \mathrm{~cm}^{-1}$ relates to Fe-O group, which indicates the presence of iron-oxide particles on PSAM hydrogel.

(Fig. 2)

\section{Adsorption Isotherm}

In addition to adsorption kinetics, the equilibrium adsorption isotherms is also one of the most important approach to understand the mechanism of the adsorption. Adsorption isotherms indicated the relationship between the mass of pesticide adsorbed per unit mass of the sorbent and the liquid phase pesticide concentration at constant temperature.

Two classic isotherm equations namely Langmuir and Freundlich were selected for this study to determine the isotherm parameters.

The Langmuir isotherm is based on the monolayer sorption of pesticide on the surface of the sorbent and is represented by the following equation -

$$
\frac{C_{e}}{C_{a d s}}=\frac{1}{Q b}+\frac{C_{e}}{Q}(8)
$$

where ' $Q$ ' is the maximum sorption capacity indicating monolayer coverage of the sorbent with sorbate while ' $\mathrm{b}$ ' is a constant related to the free energy of adsorption, independent of temperature. $\mathrm{C}_{\mathrm{e}}$ is the equilibrium pesticide concentration $\left(\mathrm{mg} \mathrm{dm}^{-3}\right)$ in aqueous solution. The values of ' $\mathrm{Q}$ ' $\left(\mathrm{mmol} \mathrm{g}^{-1}\right)$ are calculated from slope of the linear plots whereas the values of ' $\mathrm{b}$ ' are estimated from the intercepts of the plots. The value of maximum adsorption of pesticide $\mathrm{Q}$ was found to be 1.2019 at $10 \mathrm{mg} \mathrm{dm}^{-3}$ in 60 minute. The value of $\mathrm{R}^{2}$ was 0.918 which shows that the results of adsorption are better fitted in Langmuir isotherm.

\section{Freudlich isotherm}

Freudlich adsorption is the relationship between the amount of pesticide adsorbed per unit mass of the adsorbent and concentration of the pesticide at equilibrium [25]. The linearized Freudlich model can be described by the following equation:

$$
\operatorname{In} q_{e}=\left(\frac{1}{n_{f}}\right) \operatorname{In} C_{e}+\operatorname{In} K_{f}
$$

where $K_{f}$ is the Freundlich constant related to the binding energy and adsorption capacity (mg/g) and $1 / n_{F}$ is the heterogeneity factor. From the plot of $\mathrm{q}_{\mathrm{e}}$ vs. $\mathrm{C}_{\mathrm{e}}$ from experimental data, $\mathrm{K}_{\mathrm{f}}$ and $1 / \mathrm{n}_{\mathrm{f}}$ can be evaluated from the intercept and slope of the linear regression, respectively. The result are shown in figure in Table 3.

\subsection{Batch adsorption studies:}

The adsorption experiments were carried out by varying $\mathrm{pH}$, contact time, adsorbent dose and concentration. 
Synthesis and Characterization of paramagnetic (poly styrene-co-acrylamide) hydrogel: used as..

\subsubsection{Effect of pH:}

The removal of pesticide from aqueous solution by adsorption is highly dependent on the $\mathrm{pH}$ of the solution which affects the surface charge of the adsorbent and the degree of ionization. Experiments were performed at $25^{\circ} \mathrm{C}$ at $0.01 \mathrm{mg} \mathrm{dm}^{-3}$ initial pesticides concentration at varying $\mathrm{pH}$ from 2 to 7 . The effect of $\mathrm{pH}$ on pesticide adsorption is shown in figure 8 which shows that at low $\mathrm{pH}$ values, the removal percentage of pesticide was high due to the increase in positive charge density (proton) on the surface sites, resulting in electrostatic attraction between the pesticide and edge group with positive charge on the surface.

\subsubsection{Effect of concentration:}

In order to understand the absorbate and adsorbent interaction the adsorption experiments were carried out at different concentrations of pesticide solution. The initial pesticide concentration was varied in the range of 0.005 to $0.04 \mathrm{mg} \mathrm{dm}^{-3}$ and the highest uptake was found at $0.01 \mathrm{mg} \mathrm{dm}^{-3}$ concentration. Above this concentration, a decrease in the adsorbed amount was noticed which may be due to progressive saturation of binding sites.

\subsubsection{Effect of adsorbent dose:}

Adsorbent dose is an important parameter because it determines the capacity of an adsorbent for a given initial concentration of the adsorbate. The effect of adsorbent dose on the removal of pesticide was studied by varying the dose of adsorbent in the range of 0.2 to $1 \mathrm{~g}$ at fixed pesticide concentration.

\subsubsection{Effect of Time:}

The adsorption of pesticide increased with increasing contact time and became almost constant after $60 \mathrm{~min}$. From figure 10, the plot reveals that the pesticide removal percentage is higher at the beginning, which may be due to larger surface area of the adsorbent being available for adsorption.

\section{Fixed -bed adsorption dynamics}

The operation parameters such as inlet concentration $\left(\mathrm{C}_{0}\right)$, flow rate and bed height have a great influence on break through and saturation time and on the dynamics of the column. In this study, the effect of these parameters on the breakthrough curves of pesticide was investigated.

\subsection{Factors affecting the column adsorption}

\subsubsection{Effect of flow rate:}

For the purpose of investigating the effect of the flow rate on the breakthrough curves of pesticide, the column experiments were conducted at three different flow rates $(1.0,2.0$ and $3.0 \mathrm{~mL} / \mathrm{min})$, at an inlet pesticide concentration of $0.01 \mathrm{mg} \mathrm{dm}^{-3}$ and a bed height of $4 \mathrm{~cm}$. It was observed that the breakthrough time was found to decrease with the increase in flow rate. At the flow rate of $3.0 \mathrm{~mL} / \mathrm{min}$, more rapid removal of pesticide was noticed in the initial step and the rate decreased thereafter to finally reach the saturation as shown in figure 12 . At higher flow rates the saturation time of the adsorbate in the column is usually not enough for the adsorption equilibrium to be reached and the pesticide solution leaves the column before obtaining equilibrium .Hence the adsorption capacity decreased with increase in the flow rate.

\subsubsection{Effect of bed height:}

The accumulation of pesticide in a fixed-bed column is dependent on the quantity of adsorbent inside the column. Increase in bed height of adsorption columns leads to an extension of breakthrough point as well as the exhaustion time of adsorbent [24]. This is due to increase in the amount of adsorbent in column which increases in service area. In order to study the effect of bed height on the adsorption performance and the breakthrough curves, fixed -bed experiments were conducted at different bed heights $(2.0 \mathrm{~cm}, 4.0 \mathrm{~cm}$ and $6.0 \mathrm{~cm}$ ) with an inlet pesticide concentration of $0.01 \mathrm{mg} \mathrm{dm}^{-3}$ and a flow rate of $1.0 \mathrm{mg} / \mathrm{mL}$. The breakthrough time decreased with a decreasing bed height from 6 to $2 \mathrm{~cm}$, as binding sites were restricted at low bed heights. With increase in the bed height, the number of binding sites is increases, thus increasing the adsorption area of the adsorbent, hence the adsorption capacity increases.

\subsubsection{Effect of inlet pesticide concentration:}

The effect of inlet pesticide concentration on the shape of breakthrough curves was studied at different inlet concentrations (i.e. $0.01,0.02$ and $\left.0.03 \mathrm{mg} \mathrm{dm}^{-3}\right)$, at a constant flow rate $(1.0 \mathrm{ml} / \mathrm{min}$ ) and bed height of $4.0 \mathrm{~cm}$ as shown in figure 14. It was observed that when the initial pesticide concentration increased from 0.01 to $0.03 \mathrm{mg} \mathrm{dm}^{-3}$, the breakthrough time decreased because the binding sites became saturated more quickly in column. 


\subsection{BDST model:}

\section{Adsorption parameters estimation}

The breakthrough service time (BDST) model is based on measuring the capacity of the bed at various percentage breakthrough values. The BDST model constant can be helpful to scale up the process for other flow rates and concentrations without further experimentation. It is used to predict the column performance for any bed height, if data for some depths are known. It states that the bed depth $\mathrm{Z}$ and service time $\mathrm{t}$ of a column bears a linear relationship. The rate of adsorption is controlled by the surface reaction between adsorbate and the adsorbent. The BDST equation can be expressed as follows

$t=\frac{N_{0} Z}{C_{0} Q}-\frac{1}{K_{a} C_{0}} \operatorname{In}\left(\frac{C_{0}}{C_{t}}-1\right)$

where $C_{t}$ is the breakthrough pesticide concentration $(\mathrm{mg} / \mathrm{L}), \mathrm{N}_{0}$ the sorption capacity of bed (mg/L), Q the Linear velocity $(\mathrm{mg} / \mathrm{min})$ and $\mathrm{k}_{\mathrm{a}}$ is the rate constant. $\mathrm{Z}$ is depth of column bed $(\mathrm{cm})$. The high correlation coefficient value $\left(\mathrm{R}^{2}=1\right)$ indicated the validity of the BDST model for the present system.

\section{Conclusions}

We make use of the colour change of gold nano particles from red to blue by salt induced aggregation for the monitoring of the presence of pesticide. The developed method is very simple and doesn't need any expensive chemicals and the magnetic nano particles of iron oxide loaded ploy (styrene-co-acrylamide) hydrogel was synthesized and used for the effective removal of pesticide "chlorpyrifos" from aqueous solution. Batch experiment indicated that the time required to attain equilibrium was $60 \mathrm{~min}$ and the adsorption followed the Langmuir model. Maximum adsorption of pesticide was achieved within the $\mathrm{pH}$ range 3.0-4.0.The fixed bed column breakthrough curves were analyzed at different flow rates, bed heights and concentrations. The BDST model was successfully used for predicting curves by a fixed bed of Poly SAM hydrogel using different flow rates and bed heights. The super paramagnetic poly styrene-co-acrylamide hydrogel showed maximum removal of chlorpyrifos as $97 \%$, hence is a highly efficient adsorbent and may provide an alternative method for pesticide removal from contaminated water.

\section{Acknowledgment}

The authors would like to thanks to UGC-DEA consortium of Scientific Research, Indore, India for FTIR analysis. AIIMS New Delhi, India for TEM analysis.

\section{References}

[1]. H.R.Schulten, The three-dimentional structure of humic substances and soil organic matter study by computational analytical chemistry, Fresenius' $j$. of analyt. Chemi. 351, 1995, 62-73.

[2]. S.kang, D Amarasiriwardena, P. Veneman, B.s.xing, characterization of ten sequentially extracted humic acids and a human form a soil in western wassa-chusetts, soil science $168,2003,880-887$

[3]. Costa, L.G. Current issues in organophosphate toxicology. Clinica.Chimica.Acta.366, 2006 1-13.

[4]. Zeljezic, D. Vrhovac, V.G. Sister chromatid exchange and proliferative rate index in the longitudinal risk assessment of occupationalexposure to pesticides. Chemosp. ,46, 2002, 295-303,

[5]. G. Shukla, A. Kumar, M. Bhanti, P.E. Joseph, A. Taneja, Organochlorine pesticidecontamination of ground water in the city of Hyderabad, Environ. Int. 32, 2006, 244-247.

[6]. J. Cornejo, R. Celis, I. Pavlovic, M.A. Ulibarri, Interactions of pesticides withclays and layered double hydroxides: a review, Clay Miner. 43, 2008, 155-176.

[7]. $\quad$ V. Rives, Layered Double Hydroxides: Present and Future, Nova Science Publishers, Inc., New York, 2001.

[8]. Racke, K.D, Lubinnski R.N, Fontaine D.D, Miller, J.R., Mccall P.J. and Oliver G.R, Acs, Sym Ser 522, 199370.

[9]. Dyer, S.M, Cattani, M, Pisaniello,.D.L. Williams, F.M and Edwards, Journal of Toxicology 169, 2001, 177.

[10]. A. Saeed, M. IqbalBioremoval of cadmium from aqueous solution by black gram husk (Cicerarientinum) Water Research 37 , 2003,3472-34809.

[11]. S.Chowdhury, P. Saha, Sea shell powder as a new adsorbent to remove Basic Green 4 (malachite Green) from aqueous solution, Equilibrium, kinetic and thermodynamic studies J. chem, Eng. 164, 2010,168-173.

[12]. G, Annadurai, R.S, Juang, D.J, Lee Use of cellulose-based wastes for adsorption of dyes from aqueous solutions. J.Hazard. Mater. 922, 2002, 63-274.

[13]. V.K, Gupta, I, Ali Removal of endosulfan and methoxychlor from water on carbo slurry J. of Environ. Sci.Technol. 42, 2008, 766770.

[14]. V.K,Gupta, I, Ali, V.K.SainiRemoval of chlorophenols from wastewater using red mud: an aluminum industry waste J. of Environ. Sci. Technol. 38, 2004, 4012-4018.

[15]. Wu, F.C., R.L,Tseng,R.S. Adsorption of dyes and phenols from water on the activated carbons prepared from corncob wastes J. Environ. Technol.22, 2001,205-2139.

[16]. C, Xia, F, Zhou, Y, Chen, L, Zhang Removal of lead from aqueous solution by sewage sludge carbon adsorbent j. of Adv.Mater. Res. 242, 2011, 1866-1869.

[17]. J.S.Noh, J .A Schwarz (1989) Stimation of the point of zero charge of simple oxides by mass titration. J. of colloid Interface Sci.130, 1989,157-164.

[18]. K.Y. Foo, B.H. Hameed, Insights into the modeling of adsorption isotherm systems, Chem. Eng. J. 156 ,2010, 2-10. 
Synthesis and Characterization of paramagnetic (poly styrene-co-acrylamide) hydrogel: used as..

[19]. E.S. Dragan, D.F. ApopeiLoghin, Enhanced sorption of methylene blue from aqueous solutions by semi-IPN composite cryogels with anionically modified potato starch entrapped in PAAm matrix, Chem. Eng. J. 234, 2013, 211-222

[20]. A.T. Paulino, M.R. Guilherme, A.V. Reis, G.M. Campese, E.C. Muniz, J. Nozaki,Removal of methyleneblue dye from an aqueous media using superabsorbenthydrogel supported on modified polysaccharide, J. Colloid Interface Sci. 301, 2006, 55-62

[21]. P.V. Dadhaniya, M.P. Patel, R.G. Patel, Swelling and dye adsorption study ofnovel superswelling [acrylamide/Nvinylpyrrolidone/3(2-hydroxyethylcarbamoyl) acrylic acid] hydrogels, Polym. Bull. 57 2006, 21-31.

TEM

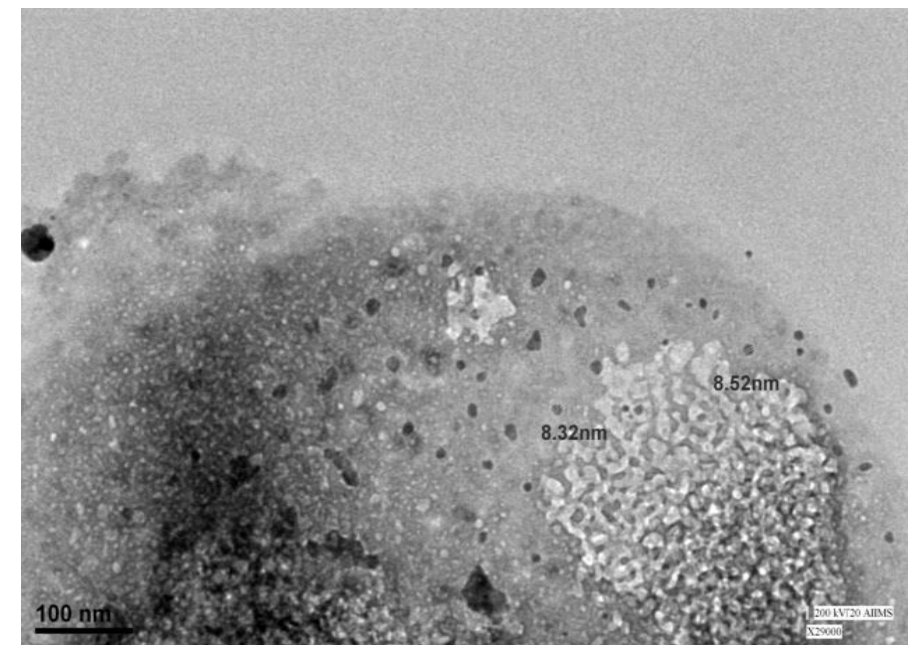

Fig.2. Transmission Electron Micrograph of iron oxide Nanoparticle

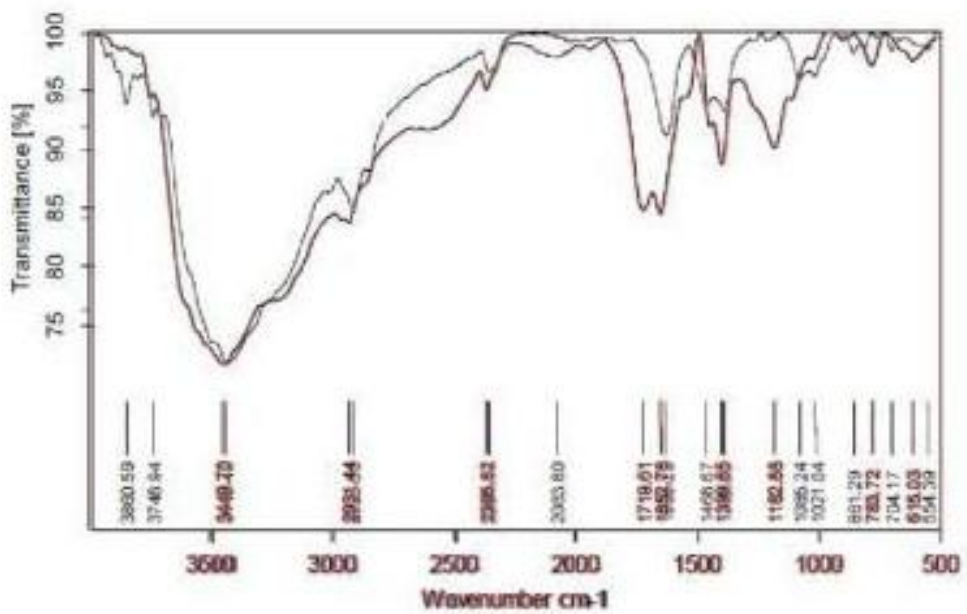

Fig. 3. FTIR Spectra pattern of paramagnetic poly (styrene-co-acrylamide) co-polymer.

Table 3 Constants of Langmuir, Freundlich isotherm models for pesticide

\begin{tabular}{|l|c|l|l|l|l|}
\hline \multicolumn{4}{|l|}{ Langmuir constant } & \multicolumn{3}{l|}{ Freundlich constant } \\
\hline $\mathrm{Q}_{0}$ & $\mathbf{b}$ & $\mathbf{R}^{\mathbf{2}}$ & $\mathbf{K}_{\mathbf{f}}$ & $\mathbf{N}$ & $\mathbf{R}^{\mathbf{2}}$ \\
\hline 1.2019 & 22.607 & 0.918 & 0.06391 & 20.408 & 0.049 \\
\hline
\end{tabular}

